\title{
Niveles de evidencia y grados de recomendación en kinesiología; una comparación entre cinco sistemas de clasificación. Estudio piloto
}

\author{
R. Aguilera-Eguía ${ }^{1}$, C. Rojas-Sepúlveda², G. Aguayo-Alcayaga² y D. Sánchez-León ${ }^{2}$ \\ ${ }^{1}$ Facultad de Ciencias de la Actividad Física. Universidad San Sebastián. Santiago de Chile. ${ }^{2}$ Kinesiología. \\ Universidad de las Américas. Santiago de Chile, Chile
}

Aguilera-Eguía R, Rojas-Sepúlveda C, Aguayo-Alcayaga G, Sánchez-León D. Niveles de evidencia y grados de recomendación en kinesiología; una comparación entre cinco sistemas de clasificación. Estudio piloto. Rev Soc Esp Dolor 2015;22(5):205-211.

\begin{abstract}
Objective: To evaluate which of the five classification systems is simpler to understand a Physical therapy evidence levels and grades of recommendation

Design: Randomized controlled trial, pilot study.

Participants: Physical therapy dedicated to musculoskeletal rehabilitation.

Intervention: Case report describing a female patient diagnosed with mild osteoarthritis of the right knee, a specific Medline search was performed to answer the following question: Can therapeutic ultrasound reduce pain and improve physical function? A systematic review that answers the question was not found. The same evidence was presented differently according to the following categories of evidence: NICE, SIGN, GRADE, EMBC and CTPHC.

Results: 8 therapists agreed to participate. A GRADE 87.5 $\%$ considered the simplest classification compared to $12.5 \%$ (other systems), with a RR $=7.0(1.090,95 \%$ CI 44.6) and Risk Difference DR $=75 \%(42.59,95 \%$ CI 107.4). To see the association showing the relationship between the exposed and unexposed GRADE classification system was used Chi2 statistical Mantel-Haenszel, with a value $\mathrm{p}=8.438$.

Conclusion: Apparently GRADE is the most simple for therapists to understand how scientific evidence.
\end{abstract}

Key words: Systematic review. Clinical trial. Randomized controlled trial. Therapy. Ultrasonic. Osteoarthritis of knee.

Recibido: 01-09-14.

Aceptado: 15-12-15

\section{RESUMEN}

Objetivo: evaluar cuál de los cinco sistemas de clasificación resulta más simple para que un kinesiólogo pueda entender los niveles de evidencia y grados de recomendación.

Diseño: estudio clínico aleatorizado, estudio piloto.

Participantes: kinesiólogos dedicados a la rehabilitación traumatológica.

Intervención: caso clínico que describía a una paciente con diagnóstico de artrosis leve de rodilla derecha. Se realiza una búsqueda específica en Medline para responder la siguiente pregunta: ¿puede el ultrasonido terapéutico disminuir el dolor y mejorar la función física? Se encontró una revisión sistemática que responde a la pregunta. La misma evidencia fue presentada de diferente forma según las siguientes clasificaciones de evidencia: NICE, SIGN, GRADE, CEBM y CTPHC.

Resultados: aceptaron participar ocho kinesiólogos. Un $87,5 \%$ consideró GRADE la clasificación más simple en comparación con un $12,5 \%$ (otros sistemas), presentando un $\mathrm{RR}=7,0(1.090,44,6$ IC95\%) y una Diferencia de Riesgo $(D R)=75 \%(42.59,107,4$ IC95\%). Para ver la asociación que presenta la relación entre los expuestos y no expuestos al sistema de clasificación GRADE se utilizó el estadístico Chi2 de Mantel-Haenszel, que presentó un valor $\mathrm{p}=8.438$.

Conclusión: al parecer GRADE es la forma más simple para que los kinesiólogos puedan entender la evidencia científica.

Palabras clave: Revisión sistemática. Ensayo clínico. Ensayo clínico aleatorizado. Terapia de ultrasonido. Osteoartritis de rodilla.

\section{INTRODUCCIÓN}

Más que nunca, estas últimas décadas se han caracterizado por un incremento enorme y exponencial de la cantidad de información biomédica publicada y por la posibilidad 
de acceso a estos canales de información (1). Sin embargo, esto que a priori parece ser una ventaja puede convertirse en un problema para los clínicos, ya que este gran volumen de información establece una necesidad profesional de actualización constante.

Lo mencionado anteriormente conlleva ciertas exigencias adicionales que hoy día el clínico debe tener en cuenta, como acceder de forma fácil y periódica a estos canales de información para poseer la capacidad de evaluar críticamente la literatura científica desde una perspectiva metodológica y extrapolar los resultados reportados por dichos estudios. Solo de esta forma se podría incorporar este nuevo conocimiento a la práctica clínica habitual.

Contrariamente a lo expuesto, algunos estudios coinciden en que los clínicos suelen tener dificultades para localizar información relevante, leer lo que se publica en inglés, analizar e interpretar los resultados de los estudios para poder aplicarlos a la atención de sus pacientes en lugares adecuados y en el tiempo oportuno (2-6). Lo cierto es que existe un desfase enorme entre la evidencia científica publicada y la práctica clínica habitual (7), ya que esta última se basa en un porcentaje muy bajo de evidencia científica, lo que además se relaciona con otros factores, como los años de egreso (8). Esta problemática se torna aún más compleja cuando al decidir revisar un tema clínico de interés nos encontramos con demasiada información, que además reporta resultados discordantes y/o contradictorios sobre la misma pregunta de investigación.

Es por este motivo que surge una nueva corriente denominada "Medicina Basada en la Evidencia (MBE)". Este es un movimiento destinado a llenar la tradicional brecha existente entre la práctica clínica y la investigación (9), promoviendo el cambio desde la toma de decisiones clínicas informadas" para ser aplicadas en un paciente "particular".

Decidir si una intervención resulta -adecuada" para un paciente determinado equivale a establecer si existe o no un grado razonable de certeza de que el balance entre los beneficios, efectos adversos y costes de dichos resultados sean lo suficientemente favorables como para que merezca la pena su aplicación.

En la toma de decisión clínica existen conceptos relevantes, como la calidad de la evidencia y la fuerza de recomendación $(10,11)$. Ambos constituyen un pilar fundamental de la práctica basada en evidencia, en su intento de "estandarizar" y proporcionar reglas a los clínicos para analizar los artículos científicos, determinar su validez y considerar su utilidad clínica (balance entre riesgos y beneficios).

Actualmente, cada vez tiene mayor importancia tomar decisiones clínicas que estén fundamentadas en el mejor nivel de evidencia (esto nos indica hasta qué punto nuestra confianza en la estimación de un efecto es adecuada para apoyar una recomendación) y en la fuerza de recomendación (indica hasta qué punto podemos confiar si poner en práctica la recomendación y si esta conlleva más beneficios que riesgos).
Si bien es cierto que los conceptos mencionados anteriormente se relacionan y complementan entre sí, estos conceptos se ocupan de aspectos totalmente distintos, aunque la fuerza de una recomendación se apoya en la calidad de la evidencia por la cual se sustenta $(12,13)$. Esto no parece ser suficiente, debido a que la magnitud del efecto sobre un outcome de interés podría presentar poca precisión o ser irrelevante desde el punto de vista clínico (outcome intermedio).

Dentro de los elementos relevantes en la toma de decisión clínica para poder recomendar con seguridad la utilización de una intervención (14) está la realización de un balance entre los "beneficios" frente a los "riesgos". Este punto no solo considera los posibles eventos adversos que pudiera presentar una intervención, también incluye la magnitud del efecto, daño, coste, valores y preferencias de los pacientes.

El primer intento confiable para introducir rigor y transparencia en la jerarquización de la evidencia fue realizado hace más de 30 años por la Canadian Task Force on Preventive Health Care (CTFPHC) (7), trabajo adaptado posteriormente por la United State Preventive Services Task Force (USPSTF) (8). Desde entonces, numerosas organizaciones e instituciones han ido desarrollando sus propios sistemas jerárquicos.

Las escalas pueden utilizar letras (A, B, C, etc.), números (I, II, III) o una combinación de ambos (Ia, IIb, IIa, etc.). A modo de ejemplo, si evaluamos el nivel de evidencia y el grado de recomendación de un ensayo clínico aleatorizado (15) (elegido azarosamente para el ejemplo) con las escalas mencionadas anteriormente encontraríamos lo siguiente: según la valoración del CTFPHC (IA); SIGN (I++A); Oxford (Ib-A); USPSTF (moderada A); NICE (I+A); Van tulder (evidencia moderada); GRADE (very low). Sin embargo, la forma de expresar los niveles de evidencia y grados de recomendación es totalmente dispar entre las escalas citadas (aun evaluando el mismo diseño metodológico, presentando diferencias en los criterios de graduación, reproducibilidad, niveles de evidencia e interpretación de los grados de recomendación), lo que puede confundir e incrementar la incertidumbre al momento de tomar una decisión clínica.

En el año 2000, con el objetivo de abordar las deficiencias de los actuales sistemas de clasificación, un grupo de clínicos y expertos en metodología crearon el grupo de trabajo GRADE, que ha elaborado una clasificación que presenta un enfoque sistemático y explícito a partir de la definición de una series de criterios necesarios para hacer juicios sobre la calidad de la evidencia y la fuerza de la recomendación.

Es por este motivo que surgió nuestra pregunta de investigación: ¿es GRADE la herramienta de clasificación más simple para entender los niveles de evidencia y grados de recomendación en comparación con NICE, SIGN, CEBM 
y CTPHC en el momento de tomar una decisión clínica? El propósito de nuestro trabajo es evaluar en cuál de los cinco sistemas de clasificación resulta más simple entender los niveles de evidencia y grados de recomendación para los kinesiólogos que trabajan en el área de rehabilitación traumatológica.

\section{MATERIALES Y MÉTODOS}

\section{Participantes}

Se invitó a participar a kinesiólogos de la clínica Santa María que no tuvieran formación en salud basada en evidencia. Los kinesiólgos debían desempeñar sus funciones clínicas en el área de rehabilitación traumatológica, en Santiago de Chile.

A cada participante se le entregó un documento de consentimiento informado en el cual se les explicaba en qué consistía el trabajo de investigación, constatando que de aceptar su participación era de forma voluntaria y no serían compensados de ninguna forma por ello. Si alguno de los profesionales rechazaba la invitación de participar en nuestro estudio se dejaba registro y se generaba una nueva convocatoria de la base de datos. Una vez aceptado y firmado el documento de consentimiento informado comenzaba la entrevista. Todas las entrevistas fueron realizadas en una atmósfera relajada, sin las distracciones de las tareas clínicas, en oficinas privadas. Este estudio fue aprobado por la facultad de Ciencias de la Salud, Escuela de kinesiologia Universidad de las Américas, Santiago de Chile.

\section{Intervenciones}

A los kinesiólogos se les entregó un caso clínico que describía a una paciente de 66 años con diagnóstico de artrosis leve de rodilla derecha, mediante el que se pretendía responder la siguiente pregunta de investigación: en sujetos con artrosis leve de rodilla, ¿puede el ultrasonido terapéutico disminuir el dolor y mejorar la función física?

Para dar respuesta a la interrogante dos autores (GA, RA) realizaron una búsqueda en la base de datos Medline, utilizando el filtro metodológico -CLINICAL QUERIES-, con la siguiente estrategia: ("Osteoarthritis, Knee" [Mesh]) AND "Ultrasonic Therapy" [Mesh].

El catastro arrojó un total de cuatro revisiones sistemáticas (17-20), dentro de las cuales una respondió de forma específica a la pregunta de investigación (17). Dos autores (GA y RA), de forma independiente, coincidieron en que la revisión sistemática de Loyola (17) respondía claramente a nuestra pregunta, la cual concluía en términos generales que "la terapia de US puede ser eficaz en la reducción de dolor y en el incremento de la función física en aquellos pacientes que presentan osteoartritis de rodilla". Tras esta búsqueda, estos autores, de forma independiente, evaluaron los niveles de evidencia y el grado de recomendación de la RS seleccionada con los distintos sistemas de clasificación (NICE, SIGN, CEBM, CTPHC y GRADE).

Otros dos autores (CR y DS) entrevistaron a los kinesiólogos que aceptaron participar en la investigación. Los participantes debían leer con atención el caso clínico presentado por los autores y escoger la clasificación de la evidencia que les resultara más simple de entender los niveles de evidencia y grados de recomendación para el uso de US terapéutico para reducir el dolor y mejorar la funcionalidad en sujetos que presentan artrosis leve de rodilla.

La clasificación GRADE, estuvo presente en todos los grupos, a diferencia de los otros sistemas de clasificación, que solo estuvieron en uno de los cuatro grupos.

\section{Aleatorización}

El proceso de aleatorización fue realizado por una estudiante de tercer año de kinesiología (BG). BG no realizó otra labor en el proyecto de investigación. La aleatorización se llevó a cabo mediante una tómbola. El autor GA estaba encargado del ocultamiento de la asignación, donde una vez finalizado el proceso de aleatorización introducía la asignación en un sobre negro que luego era sellado. CR y DS desconocían la asignación de los kinesiólogos a los sistemas de clasificación de la evidencia, motivo por el cual se encontraban ciegos en el momento de realizar las entrevistas. Los ocho kinesiólogos fueron divididos en cuatro ramas (GRADE vs. NICE; GRADE vs. SIGN; GRADE vs. CEBM; GRADE vs. CTFPHC) (Fig. 1).

\section{Resultado evaluado}

Nuestro resultado primario pretende demostrar qué sistema de clasificación resulta más simple para un kinesiólogo que trabaja en el área de la rehabilitación traumatológica para entender la evidencia científica en el momento de tomar una decisión clínica. En nuestro estudio consideraremos el término -entender- como lo define la Real Academia Española: "comprender, entender el sentido de algo".

Para esto se entregó un caso clínico en el que se reportaban los resultados de interés contenidos en una revisión sistemática (dolor y función) expresados mediante la clasificación perteneciente a los siguientes sistemas: GRADE, NICE, SIGN, CEBM y CTFPHC. Los participantes debían escoger una de las dos clasificaciones expuestas (GRADE o NICE; GRADE o SIGN, y así sucesivamente) y marcar con una cruz la alternativa en la que les resultaba más fácil entender los resultados mencionados anteriormente. 


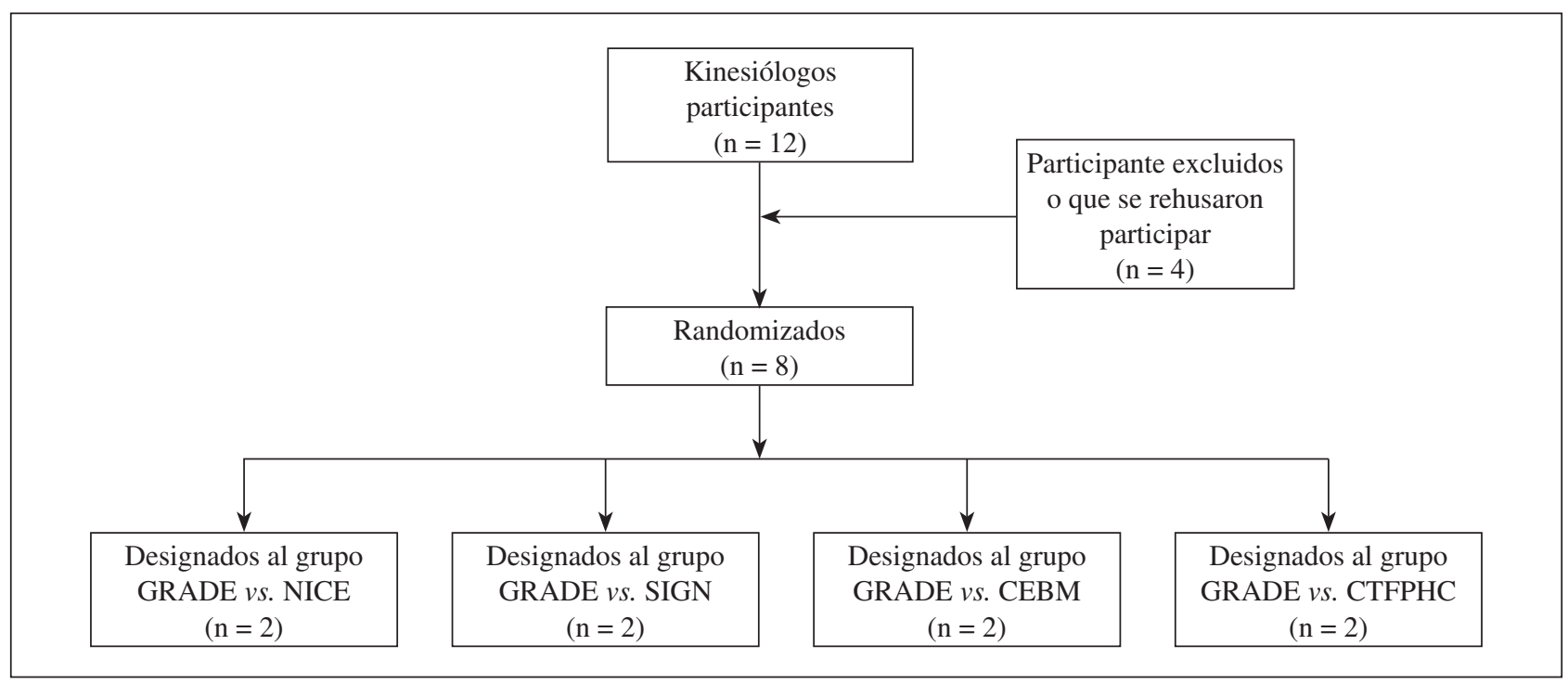

Fig. 1. Diagrama sobre el flujo de participantes

Nuestro desenlace pertenece a una variable dicotómica (GRADE u otro sistema de clasificación), por lo tanto expresaremos sus valores en riesgo relativo (RR) y diferencia de riesgo (DR), con sus respectivos intervalos de confianza (IC 95\%).

Para evitar sesgo nos preocupamos de que los clínicos que participaran en nuestro trabajo de investigación no tuvieran formación en medicina basada en evidencia ni conocimientos en los distintos sistemas de clasificación de la evidencia.

\section{Análisis estadístico}

Para el análisis estadístico de las variables dicotómicas empleadas en nuestro estudio se utilizó el software OpenEpi versión 3.01 (21), mediante el que analizamos los datos en una tabla de contingencia simple ( $2 \times 2)$. Para poder observar su asociación se utilizó el estadístico de $\chi^{2}$ de Mantel-Haenszel (Tabla II).

\section{RESULTADOS}

De los doce kinesiólogos que fueron invitados a particiar en este estudio, cuatro negaron su participación, quedando un total de ocho kinesiólogos que desempeñan su labor clínica en el área de la rehabilitación traumatológica, quienes fueron distribuidos por azar en cuatro grupos (Fig. 1).

En relación con las preferencias reportadas por los kinesiólogos frente a los distintos sistemas de clasificación, encontramos que un $87,5 \%$ frente a un $12,5 \%$ consideró que el sistema GRADE le resultó más simple para poder entender la evidencia en el momento de tomar una desición clínica comparado a los otros sitemas respectivamente, presentando un riesgo relativo $\mathrm{RR}=7,0$ (1.090, 44,6 IC 95\%) y una diferencia de riesgo $\mathrm{DR}=75 \%(42,59,107,4 \mathrm{IC}$ 95\%). Para ver la asociación que presenta la relación entre los expuestos y no expuestos al sistema de clasificación GRADE se utilizó el estadístico Chi de Mantel-Haenszel, presentando un valor $\mathrm{p}=8.438$ (Tabla II).

\section{DISCUSIÓN Y CONCLUSIÓN}

En el momento de enfrentarnos a un paciente y decidir qué intervenciones vamos a utilizar debemos considerar siempre la mejor opción disponible a nuestro alcance; esto se traduce en -la mejor evidencia disponible-. Es fundamental recordar que la mejor evidencia que existe va a depender de nuestra pregunta de investigación, si queremos saber sobre tratamientos la mejor evidencia corresponde a revisiones sistemáticas o metaanálisis de estudios clínicos aleatorizados o estudios clínicos aleatorizados (siempre y cuando no exista una RS que evalúe el tema considerado).

La medicina basada en evidencia (MBE) en la toma de desición clínica tiene un rol fundamental, debido a que surgió principalmente para llenar la tradicional brecha existente entre la -Práctica clínica y la investigación" (22), promoviendo el cambio desde la toma de decisiones clínicas "informadas" para ser aplicadas en un paciente "particular".

La MBE se define como "el uso explícito, racional y juicioso de la mejor evidencia disponible en la toma de decisiones clínicas, incorporando los valores y las preferencias de los pacientes- (22). Actualmente la MBE se estructura 
TABLA I. CARACTERÍSTICAS DE LA LÍNEA DE BASE

\begin{tabular}{|c|c|c|c|c|}
\hline & GRADE vs. NICE & GRADE vs. SING & GRADE vs. CEBM & GRADE vs. CTFPHC \\
\hline Edad (DS) & $28(2)$ & $36,5(16)$ & $26,5(2)$ & $35,5(7)$ \\
\hline Años de experiencia clínica (DS) & $5(8)$ & $13,5(13)$ & $4,5(2,1)$ & $14(7)$ \\
\hline \multicolumn{5}{|l|}{ Género } \\
\hline Femenino & 2 & 1 & 1 & 0 \\
\hline Masculino & 0 & 1 & 1 & 2 \\
\hline Formación en SBE & -- & -- & -- & -- \\
\hline
\end{tabular}

Edad: expresada en promedio; DS: desviación estándar; GRADE: clasificación de la evaluación, desarrollo y valoración de las recomendaciones; NICE: Instituto Nacional para la Salud y la Excelencia Clínica; SIGN: directrices de la red interescolar escocesa; CEBM: Centro de Medicina Basada en Evidencia de Oxford; CTFPHC: Grupo de trabajo canadiense sobre el cuidado de la salud preventiva; años de experiencia clínica: expresados en promedio.

TABLA II. RESULTADOS

\begin{tabular}{lcccc}
\hline \multicolumn{5}{c}{ ESTADÍSTICAS DE LA TABLA 2 X 2} \\
\hline \multicolumn{5}{c}{ Análisis de tabla simple } \\
\hline \multicolumn{5}{c}{ Clasificación } \\
\hline \multirow{4}{*}{ Entiende } & Sí & 7 & 1 & 8 \\
& No & 1 & 7 & 8 \\
& & 8 & 8 & 16 \\
\hline
\end{tabular}

bajo tres pilares fundamentales (experiencia clínica, evidencia científica y valores y preferencias de los pacientes); cada uno de estos puntos será explicado a continuación.

- Experiencia clínica: debemos reconocer que a través del tiempo la -experiencia clínica- u -opinión de expertos- ha sido una guía en la formación de muchos profesionales en el área de la salud, definiéndose -experiencia clínica- como el "dominio creciente del conocimiento y juicio que cada clínico obtiene a través de su práctica diaria" (23).

\section{MEDIDAS DE ASOCIACIÓN EXACTAS Y CHI}

\begin{tabular}{lccc}
\multicolumn{1}{c}{ Prueba } & Valor & Valor (1-cola) & Valor (2-cola) \\
\hline $\mathrm{Chi}^{2}$ sin corrección & 9 & 0,001350 & 0,002700 \\
$\mathrm{Chi}^{2}$ corregida de Yates & 6,25 & 0,006210 & 0,01242 \\
$\mathrm{Chi}^{2}$ de Mantel-Haenszel & 8,438 & 0,001838 & 0,003676 \\
Exacto de Fisher & & 0,005051 & 0,01010 \\
Mid-p exacto & & 0,002564 & 0,005128 \\
\hline
\end{tabular}

ESTIMADOR BASADO EN EL RIESGO* E INTERVALOS DE CONFIANZA AL 95\% (NO VÁLIDO PARA ESTUDIOS DE CASOS-CONTROL)

\begin{tabular}{lccc}
\hline \multicolumn{1}{c}{ Cálculos de puntos } & \multicolumn{2}{c}{ Límites de confianza } \\
\hline Ripo & Valor & Inferior-superior & Tipo \\
\hline Riesgo en expuestos & $87,5 \%$ & $50,79-99,89$ & Series de Taylor \\
Riesgo en no expuestos & $12,5 \%$ & $0,1137-49,21$ & Series de Taylor \\
Riesgo total & $50 \%$ & $28-72$ & Series de Taylor \\
Razón de riesgo & 7 & $1,099-44,61$ & Series de Taylor \\
Diferencia de riesgo & $75 \%$ & $42,59-107,40$ & Series de Taylor \\
Fracción etiológica en pob. (FEP) & $75 \%$ & $33,46-100$ & \\
Fracción etiológica en expuestos (FEE) & $85,71 \%$ & $8,974-97,76$ & \\
\hline
\end{tabular}


- Evidencia científica: es un punto fundamental en la formación de cualquier profesional de la salud, debido a que otorga los fundamentos científicos -sólidosa la experiencia clínica, es decir, aporta un mayor grado de "confianza" en el momento de tomar una decisión clínica. El objetivo de este punto es orientar al clínico para que pueda utilizar la "mejor" información científica frente a una incertidumbre clínica (24).

- Valores y preferencias de los pacientes: se entiende por "valores y preferencias de los pacientes" las "expectativas y perspectivas" que adquieren en el momento de recibir una intervención. Si pretendemos ser usuarios de la MBE no debemos olvidar que lo más importante en este proceso es respetar a nuestros pacientes, considerando no solo el riesgo-beneficio o coste-efectividad de la intervención, también debemos tener presente sus creencias, cultura, etc., en el momento de tomar una decisión clínica (25).

Si consideramos que hoy en día existe gran cantidad de información biomédica publicada y que se encuentra al alcance de todos gracias a Internet, debemos ser conscientes de que esto puede presentar efectos positivos y negativos a la vez. Es fundamental recordar que no todo lo que está publicado en inglés o en una revista de renombre es sinónimo de calidad. Por lo tanto, este fenómeno de -infoxicación-, como se ha denominado al gran auge que han presentado las publicaciones científicas, exige a los kinesiólogos y/o profesionales de la salud adquirir habilidades en la lectura crítica de artículos y la búsqueda de información científica. También debemos reconocer que existen ciertas barreras (internas y extermas) que pueden afectar al clínico cuando debe tomar una decisión clínica. Dentro de estas barreras encontramos la gran disparidad existente entre las distintas escalas de clasificación de la evidencia, lo que genera mayor ambiente de incertidumbre cuando el clínico debe tomar una decisión.

Las revisiones sistemáticas con y sin metaanálisis realizan un resumen de forma exhaustiva de toda la información disponible. Este diseño metodológico está orientado a responder una pregunta clínica específica que pudo haber sido propiciada por la práctica clínica diaria, entre otras fuentes. Sin embargo, la gran mayoría de los autores de revisiones sistemáticas no tienen claro cómo resumir la evidencia en un formato simple y que sea comprensible para todos, ya sea un estudiante de pregrado, postgrado, clínico, docente o investigador que no presenten formación en salud basada en evidencia o en análisis crítico de la literatura científica. Esto también ocurre en el momento de plasmar los resultados en las guías de práctica clínica (GPC). Actualmente existe una gran disparidad e inconsistencia en el momento de expresar las recomendaciones en las GPC, lo que genera mayor incertidumbre para el clínico en el momento de tomar una decisión (26).

Nuestro estudio piloto demostró que a los kinesiólogos les resulta más simple entender la evidencia cuando se encuentra expresada mediante la clasificación GRADE. En la búsqueda de la explicación de esta situación, creemos que GRADE, por su presentación visual, puede incrementar las posibilidades de comprensión en el momento de tomar una desición clínica. También consideramos fundamental que el sistema de clasificación GRADE presente una recomendación al final. En nuestro caso clínico fue -baja evidencia", lo que quiere decir que "la estimación del efecto no es confiable-.

Nuestro trabajo presenta algunas limitaciones, las cuales describiremos a continuación: nuestra población incluyó solo un grupo pequeño de kinesiólogos $(\mathrm{n}=8)$, todos pertenecientes al mismo centro clínico, por lo que esto podría afectar su validez externa.

Nosotros no medimos el conocimiento previo de los kinesiólogos que aceptaron participar en nuestra investigación. No se obtuvo información sobre los sujetos que se negaron a participar, ya que consideramos que son pocos para que influyeran en nuestros resultados.

La presente investigación se basa en un caso clínico de un paciente por escrito que no incluye la historia del paciente ni otros factores que podrían influir en la toma de desición clínica. Tambien debemos considerar que no incluimos todos los sistemas de clasificación existentes, debido a que son demasiados (solo incluimos los más utilizados).

A nuestro parecer, este es el primer estudio para analizar qué sistema de clasificación resulta más simple para que un kinesiólogo pueda entender la evidencia reportada por una revisión sistemática.

El sistema de clasificación GRADE demostró ser la forma más simple para que los kinesiólogos puedan entender la evidencia científica, presentando un $\mathrm{RR}=7,0$ (1.09, 44,6; IC 95\%), lo que en términos simples significa que el riesgo de entender la evidencia expresada por la clasificación GRADE por un kinesiólogo es de un 70\% comparado con las clasificaciones NICE, SIGN, CEBM, CTPHC, consideando que los profesionales de la salud incluidos en nuestro estudio no presentaban formación en salud basada en evidencia ni conocimientos previos en los sistemas de clasificación. El término -riesgo- se define de la siguiente forma: "es una combinación de la probabilidad de que se produzca un evento, el cual puede ser positivo o negativo". Por lo tanto, para nuestro estudio la palabra "riesgo" no se debe entender como algo negativo.

Como grupo de investigación, somos concientes de la gran necesidad de investigación científica que se requiere en esta área a grandes escalas, considerando otras especialidades kinésicas y más sistemas de clasificación. Es por este motivo que ya nos encontramos realizando otro estudio clínico aleatorizado considerando un tamaño muestral óptimo para poder encontrar las diferencias estadísticamente significativas entre las comparaciones de los diferentes sistemas de clasificación. 
Creemos que el número actual de sistemas de clasificación genera bastante confusión y posiblemente produzca una baja adherencia a las recomendaciones.

\section{AGRADECIMIENTOS}

Agradecemos a R. A. Rojas y Mabel E. Fredes su constante apoyo.

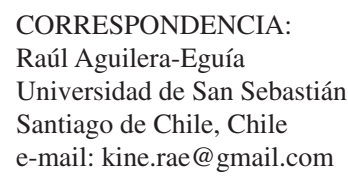

\section{BIBLIOGRAFÍA}

1. Davidoff F, Haynes B, Sackett D, Smith R. Evidence based medicine: a new journal to help doctors identify the information they need. BMJ 1995;310:1085-6.

2. EmparanzaKnorr J. Medicina Basada en Evidencia: Un aprendizaje imprescindible; An Esp Pediatr 2001;55:397-9.

3. Sackett DL, Strauss SE, Richardson WS, Rosemberg W, Haynes RB. Evidence-based medicine.How to practice and teach EBM. Edinburg; Churchill Livingstone; 2000.

4. Hayne RB. Using informatics principles and tools to harness research evidence for patient care: evidence-based informatics. Stud Health Technol Inform 1998;52 Pt 1:Suppl 33-6.

5. Rada G, Letelier LM, ¿Podemos mantenernos actualizados en medicina en el siglo XXI?; Rev Med Chile 2009;137:701-08.

6. Letelier LM, Zamarin N, Andrade M, Gabrielli L, Caiozzi G, Viviani P, et al. Exploring language barriers to Evidence-based Health Care (EBHC) in post-graduate medical students: a randomized trial. EducHealth (Abingdon) 2007;20:82-7.

7. West S, King V, Carey TS, Lohr KN, McKey N, Sutton SF, et al. Systems to rate strength of scientific evidence. AHRQ 2002;47:1-11.

8. González de Dios J. Níveles de evidencia y fuerza delas recomendaciones: necesidad de homogenización. Espacio Asma 2010;3:24-8.

9. González de Dios J, Ochoa Sangrador C. De la evidencia a la recomendación: una tarea pendiente. Med Clin 2011;135:601-2.

10. Holger JS, Best D, Vist G, Andrew DO, for the GRADE working group. Letters, numbers, symbols and words: how to communicate grades of evidence and recommendations. CMAJ 2003;169:677-80.
11. Atkins D, Best D, Briss PA, Eccles M, Falckytter Y, Flottorp $\mathrm{S}$, et al; Grade working group. Grading quality of evidence and strength of recommendations. BMJ 2004;328:1490-4.

12. Van Tulder M, Furlan A, Bombardier C, Bouter L, Editorial Board of the Cochrane Collaboration Back Review Group. Updated method guidelines for systematic reviews in the cochrane collaboration back review group. Spine 2003;28:1290-9.

13. Martín Muñoz P, González de Dios J. Valoración de la calidad de evidencia y fuerza de recomendaciones (I). Evapediatr 2010;6:63

14. Martín Muñoz P, González de Dios J. Valoración de la calidad de evidencia y fuerza de recomendaciones (II). Consideraciones prácticas en la aplicación del sistema GRADE. Evapediatr 2010;6:91.

15. Nynke S, van der windt D, Assendelft WJ, Deville WL, Korthals-de bos IB, Bouter LM. Corticosteroid injections, physiotherapy, or a wait-and-see policy for lateral epicondylitis: a randomised controlled trial. Lancet 2002;359:65762.

16. Gutiérrez Espinoza H, Aguilera Eguía R, Durán Indo M, Marros Navarro C, Sánchez Caamaño O. Revisión sistemática: uso del Brace en la epicondilalgía lateral. Fisioterapia 2011;33:217-26.

17. Loyola Sánchez A, Richardson J, Mclntyre NJ. Efficacy of ultrasound therapy for the management of knee osteoarthritis: a systematic review with meta-analysis. Osteoarthritis and Cartilage 2010;18;1117-26.

18. Rutjes AW, Nüesch E, Sterchi R, Jüni P. Therapeutic ultrasound for osteoarthritis of the knee or hip. Cochrane Database Syst Rev 2010;(1):CD003132.

19. Harris GR, Susman JL. Managing musculoskeletal complaints with rehabilitation therapy: summary of the Philadelphia Panel evidence-based clinical practice. J Fam Pract 2002;(12);1042-6.

20. Welch V, Brosseau L, Peterson J, Shea B, Tugwell P, Wells G. Therapeutic ultrasound for osteoarthritis of the knee. Cochrane Database Syst Rev 2001;(3):CD003132.

21. Dean AG, Sullivan KM, Soe MM. OpenEpi: Open Source Epidemiologic Statistics for Public Health, Versión. www. OpenEpi.com.

22. Sackett D, Rosenberg W, Gray J, et al. Evidence based medicine: what it is and what it isn't. BMJ 1996;312:71-2.

23. Bonfill X. Medicina basada en la evidencia. En: Conferencia Medicina Basada en la Evidencia. Microcine de la Facultad de Ciencias Médicas de la UNLP; 1998.

24. Guerra L. La medicina basada en la evidencia: un intento de acercar la ciencia al arte de la práctica clínica. Med Clin (Barc) 1996;107:377-82.

25. Guyatt G, Rennier.D. User's guide to the medical literature, a manual for evidence based clinical practice. Chicago, IL: American Medical Association; 2002.

26. Schünemann HJ, Fretheim A, Oxman AD. Improving the use of research evidence in guideline development: 9. Grading evidence and recommendations. Health Res Policy Syst 2006;4:21. 